\title{
PRODUÇÃO DE GELATINA DE PELE E OSSOS DE BAGRE (Clarias gariepinus)
}

\section{GELATIN PRODUCTION OF SKIN AND BONES CATFISH (Clarias gariepinus)}

\author{
Fabíola Carina Biluca ${ }^{1}$, Carline Marquetti ${ }^{2,}$ Alexandre da Trindade Alfaro ${ }^{3}$ \\ ${ }^{1,2,3}$ Universidade Tecnológica Federal do Paraná-UTFPR- Francisco Beltrão- Brasil \\ fabii_biluca@hotmail.com
}

\begin{abstract}
Resumo
O presente estudo teve como objetivo produzir gelatinas de pele e ossos de bagre (Clarias gariepinus) e determinar a viscosidade, ponto de fusão, rendimento do processo e pH das gelatinas extraídas. Para a obtenção das gelatinas, as matérias-primas foram submetidas a pré-tratamentos alcalino e ácido, com posterior extração por $12 \mathrm{~h}$ a $45^{\circ} \mathrm{C}$. A viscosidade da gelatina de pele foi 1,13 cP e ossos 0,66 cP. $O$ valor do ponto de fusão da gelatina de pele foi de $22,16{ }^{\circ} \mathrm{C}$ enquanto para gelatina de ossos $21{ }^{\circ} \mathrm{C}$. A gelatina de pele apresentou um rendimento de processo $(5,85 \%)$ consideravelmente maior que a de ossos $(1,18 \%)$. $O$ valor de $\mathrm{pH}$ das gelatinas foi semelhante.
\end{abstract}

Palavras-chave: Clarias gariepinus; pele; ossos; gelatina.

\section{Introdução}

A gelatina é um polipeptídio obtido pela degradação hidrolítica parcial de ossos, peles e colágeno, principal componente do tecido conjuntivo de animais (GIMÉNEZ, GOMÉZ-GUILLÉN e MONTERO, 2004). Todos os tipos de gelatina possuem composição similar, ou seja, água, pequena quantidade de sais minerais e proteína de tecido conectivo pura.

Segundo IBAMA (2008) a região Sul produziu 62.823,5 t de pescado em 2006 contribuindo com a maior parcela na produção nacional com 32,9\%. Os subprodutos separados durante o processamento de peixes chegam em 50-70\% da massa de matéria-prima (WANG et al., 2008), e cerca de 30\% consistem em cabeças, carcaças, pele, vísceras (KOLODZIEJSKA et al., 2009).

Neste contexto observa-se grande quantidade de subprodutos, contendo elevada quantidade de colágeno, os quais podem ser utilizados para a manufatura de gelatina. Essa proteína pode ser obtida por meio de lavagens ácida ou básica, resultando na hidrólise de colágeno (KOLODZIEJSKA et al., 2009). As propriedades e capacidade de geleificação da gelatina 
envolvem a parcial renaturação e desnaturação das suas moléculas, e suas características dependem amplamente do colágeno utilizado (BADII e HOWELL, 2006).

Além do aproveitamento de subprodutos do pescado na indústria de transformação, a getatina de pescado por muitas razões socioculturais torna-se fonte alternativa, pois não estão associadas a riscos de encefalopatia espongiforme bovina (BSE) e febre aftosa (FA), que provem de mamíferos, principalmente de suinos e bovinos das quais a maioria das gelatinas comerciais são derivadas (BADII e HOWELL, 2006; GIMÉNEZ, GOMÉZ-GUILLÉN e MONTERO, 2004).

A extração de gelatina vem sendo realizada com peles e ossos de pescados de água fria (bacalhau, pescada, salmão, entre outros) e de água quente (atum, bagre, tilápia, entre outros). Os procedimentos utilizados para produzir gelatina de pescado normalmente envolvem um prévio tratamento químico da matéria-prima, e temperatura branda durante o processo de extração (KARIM e BHAT, 2009).

O objetivo do presente estudo é a produção de gelatina de pele e ossos de bagre (Clarias gariepinus), e a determinação da viscosidade, ponto de fusão, rendimento do processo e pH das gelatinas extraídas.

\section{Material e Métodos}

\section{Material}

Peles frescas e carcaças de bagre africano (Clarias gariepinus) recém filetadas, foram coletadas, lavadas em água corrente para retirada de impurezas grosseiras e estocadas a $-18{ }^{\circ} \mathrm{C}$ até sua utilização. Todos os reagentes que foram utilizados são de grau analítico (P.A.).

\section{Pré-tratamento da matéria-prima}

\section{Extração da gelatina de pele de bagre}

As peles foram lavadas em água corrente para retirada do material superficial aderido e cortadas em peças de aproximadamente de $4 \mathrm{~cm}$ x $4 \mathrm{~cm}$. Então, o material foi imerso em solução salina de $\mathrm{NaCl} 0,2 \%(\mathrm{p} / \mathrm{v})$ por 5 minutos sob agitação contínua.

As peles cortadas foram, então, submetidas a tratamento alcalino 1:10 (p/v), em solução de $\mathrm{NaOH}$ 0,3\% (p/v), por período de 80 minutos a temperatura ambiente. Após esse procedimento, foram lavadas em água corrente para retirada do álcali em excesso, até pH abaixo de 8. A seguir, as peles foram submetidas a tratamento ácido 1:10 (p/v), em solução de $\mathrm{H}_{2} \mathrm{SO}_{4}$ 0,3\% (p/v), por 80 
minutos; e, posteriormente, lavadas em água corrente até $\mathrm{pH}$ próximo à neutralidade. As peles foram então submetidas a um terceiro tratamento em solução de acido cítrico $0,7 \%$ (p/v) em proporção de 1:10 (p/v), por 80 minutos, e lavadas em água corrente até $\mathrm{pH}$ próximo a neutralidade.

A extração da gelatina foi realizada em béquer de $1000 \mathrm{~mL}$ contendo água deionizada $(2 \mathrm{~mL}$ água : $1 \mathrm{~g}$ de pele) em banho-maria por 12 horas à temperatura de $45^{\circ} \mathrm{C}$. Após a extração, o material foi filtrado em funil de Büchner com papel de filtro Whatman $n^{\circ} 4$, fracionado em potes de polietileno hermeticamente fechados e armazenados $-18^{\circ} \mathrm{C}$.

\section{Extração de gelatina de ossos de bagre}

Os ossos foram moídos na granulométrica de $4 \mathrm{~mm}$, sendo então, lavados em água corrente para a retirada de impurezas. Os ossos moídos, foram submetidos a tratamento alcalino, em soluções de $\mathrm{NaOH}$ 0,1 M (1:2 p/v) por períodos de 24 horas em temperatura de $8{ }^{\circ} \mathrm{C}$. O material foi centrifugado e o procedimento foi repetido. Os ossos então foram lavados em água corrente para a retirada do álcali em excesso, ate $\mathrm{pH}$ próximo a neutralidade.

Os ossos moídos foram submetidos a tratamento ácido em tratamento de $\mathrm{HCl} 1 \mathrm{M}(1: 5 \mathrm{p} / \mathrm{v})$ por 24 horas em temperatura de $8{ }^{\circ} \mathrm{C}$. O material foi centrifugado e o procedimento repetido. A osseína (ossos desmineralizados) foi lavada em água corrente com o auxilio de peneira para a retirada do acido em excesso, ate $\mathrm{pH}$ acima de 4. Após, realizou-se a extração em duas etapas de 60 minutos a temperatura de 60 e $80{ }^{\circ} \mathrm{C}$, em água destilada com pH 2 e 4, ajustado pela adição de acido fosfórico mantendo a proporção de 2,5 de solução para 1 de osseína. Após a extração, o material foi filtrado em funil de Büchner com papel de filtro Whatman $n{ }^{\circ} 4$, fracionado em potes de polietileno hermeticamente fechados e armazenados $-18^{\circ} \mathrm{C}$.

\section{Composição da matéria-prima}

Foi determinada a composição centesimal para a matéria prima (peles de bagre).

Todas as determinações foram realizadas de acordo com as metodologias da A.O.A.C. 1995. $\mathrm{O}$ teor de umidade e cinzas foi determinado por gravimetria $\left(\mathrm{n}^{\circ} .950 .46\right.$ e $\mathrm{n}^{\circ} .920 .153$ respectivamente); o nitrogênio total foi determinado pela metodologia Kjeldahl ( $\left.\mathrm{n}^{\circ} .928 .08\right)$, sendo obtido o total de proteína bruta multiplicando o valor do nitrogênio total por 5,5 (fator de conversão de nitrogênio total em proteína bruta). O teor de lipídeos foi analisado através do método de extração de Sohxlet (nº 991.36). 


\section{Rendimento}

O rendimento foi determinado segundo método descrito por Yang et al., (2008) . A concentração da solução de gelatina foi determinada em refratômetro de Abbe Escala de Refração 1,300 - 1,720 nD e 95\% Brix, Marca Biobrix, Modelo 2 WAJ., e o rendimento calculado segundo a equação 1:

$$
\mathrm{R}=\underline{\mathrm{C}_{\text {gelatina }}} \cdot \underline{\mathrm{V}}_{\text {solução }} \quad \text { Equação 1 }
$$

Onde R é o rendimento em gelatina ( $\mathrm{g}$ gelatina $/ 100 \mathrm{~g}$ pele/ossos), $\mathrm{C}$ gelatina é a concentração de

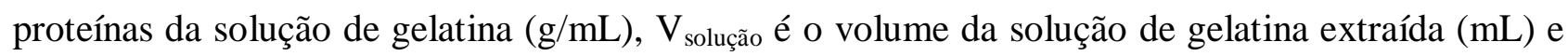
$\mathrm{M}_{\text {pele/ossos }}$ é a massa da matéria prima (g).

$p H$

Para determinação do pH foi utilizado o método da British Standard Institution (B.S.I., 1975). Foi preparada uma solução $1 \%$ (p/v) de gelatina em água destilada a $60{ }^{\circ} \mathrm{C}$, sob agitação mecânica constante, por 30 minutos; sendo após, resfriada até temperatura ambiente, aproximadamente $25^{\circ} \mathrm{C}$. A determinação do $\mathrm{pH}$ foi realizada em aparelho medidor de $\mathrm{pH}$ ORP/Temperatura-mod H.I222.

\section{Viscosidade}

A determinação da viscosidade foi feita de acordo com a metodologia descrita por Yang et. al. (2008). Água destilada foi utilizada para ajustar a concentração da solução de gelatina para 3,3\%, e as amostras foram transferidas para um viscosímetro capilar Cannon-Fenske ( $\left.\mathrm{n}^{\mathrm{o}} 100\right)$. O viscosímetro foi colocado em banho a $60{ }^{\circ} \mathrm{C}$ e aguardou-se 10 minutos até a estabilização da temperatura. Após, o tempo de escoamento foi determinado, sendo essa medida realizada em triplicata. A viscosidade foi calculada segundo a equação 2:

$$
\mu=\text { t. K. } \rho \quad \text { Equação } 2
$$


Onde $\mu$ é a viscosidade da solução de gelatina (cP) a $60^{\circ} \mathrm{C}$, t é o tempo de escoamento (s), $\mathrm{K}$ é a constante do viscosímetro (cSt/s) e $\rho$ é a massa específica da solução $(\mathrm{g} / \mathrm{mL})$.

\section{Determinação do ponto de fusão}

A determinação do ponto de fusão foi baseada na metodologia apresentada por Choi e Regenstein (2000). Alíquotas de $5 \mathrm{~mL}$ de soluções de gelatina 3,3\% foram transferidas para tubos de ensaio, e aquecidas em banho-maria a $60{ }^{\circ} \mathrm{C}$ por $15 \mathrm{~min}$. Posteriormente, os tubos foram resfriados em banho de gelo e armazenados a $10^{\circ} \mathrm{C}$ em refrigerador por $17 \pm 1 \mathrm{~h}$. Para determinação do ponto de fusão, foram adicionadas cinco gotas de indicador (75\% de clorofórmio e $25 \%$ de corante azul de metileno) sobre o gel. Os tubos foram colocados em banho-maria termostatizado a $15^{\circ} \mathrm{C}$, sendo aquecido $0,5^{\circ} \mathrm{C}$ a cada 5 minutos até atingir a temperatura de $30^{\circ} \mathrm{C}$. O ponto de fusão foi determinado, em triplicata, no momento em que as gotas do indicador começaram a se mover para o interior do gel.

\section{Resultados e Discussão}

\section{Composição centesimal da pele de bagre}

A tabela 1 apresenta a composição centesimal para a pele de bagre africano. Observa-se que o teor médio de umidade da pele foi de 74,76\%, sendo semelhante aos valores já descritos para peles de outras espécies de pescado, como a tilápia (72,6\%) (ALFARO et al., 2009).

O percentual de proteína em base úmida, encontrado na pele de bagre foi de 17,98\%, sendo inferior aos valores descritos para peles de tilápias jovens (20,3\%) e adultas (21,6\%), por Muyonga, Cole e Duodu (2004). É importante observar que o teor de proteína presente na matéria prima representará a quantidade de colágeno presente na gelatina e consequentemente seu rendimento.

O teor de gordura encontrado foi bastante elevado (6,65\%). Portanto, é necessário que os pré-tratamentos realizados na matéria prima garantam maior eficiência na eliminação do teor de lipídeos. Segundo Haug, Draget e Smidsrod (2004) o odor característico da pele do pescado se deve a compostos nitrogenados e às gorduras.

O valor de cinzas foi de $0,49 \%$ mostrando-se semelhantes aos valores descritos na literatura para a espécie do bagre africano (SOUZA et al, 1999).

Tabela 1 - Composição centesimal da pele de bagre africano.

\begin{tabular}{cc}
\hline Componentes \% & Pele de bagre \\
\hline Umidade & $74,76 \pm 0,53$ \\
Gordura & $6,65 \pm 1,1$ \\
Proteína & $17,98 \pm 1,2$ \\
Cinzas & $0,49 \pm 0,89$ \\
\hline
\end{tabular}

Valores médios referentes a três determinações \pm desvio padrão. 


\section{Rendimento}

O rendimento médio da gelatina de pele de bagre africano foi de 5,85\%. Esse valor é semelhante aos valores relatados por Jamilah e Harvinder (2002) para gelatinas de pele de tilápia negra e vermelha, que foram de 5,39\% e 7,81\%. No entanto, o rendimento para a gelatina extraída de ossos foi consideravelmente menor $(1,18 \%)$. O rendimento para a gelatina extraída de ossos de bagre africano foi semelhante aos apontados por Bandeira (2009), para a primeira extração da fração óssea de cabeça de carpa 1,2 \% que se subdividiu em 4 etapas de extração atingindo um total de 4,1\%. No entanto, Alfaro et al. (2009) descreveu rendimentos entre 6,3-8,2 para gelatinas extraídas de ossos de pescada-foguete (Macrodon ancylodon).

É possível observar diferença entre os rendimentos da gelatina extraída de pele e a gelatina extraída dos ossos, isso pode ser explicado devido aos diferentes de tratamento, onde para a extração de gelatina de ossos o procedimento é mais rigoroso.

$$
p H
$$

$\mathrm{O}$ valor médio de $\mathrm{pH}$, determinado na gelatina de pele de bagre foi de 3,20. Esse valor de pH é semelhante aos valores descritos por Jamilah e Harvinder (2002), que relataram valores de pH para gelatinas de pele de tilápia negra $(3,81)$ e vermelha $(3,05)$. Já, a gelatina de ossos apresentou valor de $\mathrm{pH}$ de 2,98, estando abaixo dos valores relatados para gelatina de ossos de pescada-foguete (Macrodon ancylodon) descritos por Alfaro (2004), que foram 3,85-4,38. Sabe-se que o pH da solução de gelatina é afetado pelo tratamento químico empregado durante a etapa de extração (GUDMUNDSSON e HAFSTEINSSON, 1997). Deste modo o pH mais elevado demonstra uma melhor eficiência das etapas de lavagem subsequientes aos tratamentos químicos durante a preparação das peles, antes da etapa de extração.

\section{Viscosidade e ponto de fusão}

Sabe-se que a viscosidade das soluções de gelatina são influenciadas por sua distribuição de pesos moleculares, onde maiores pesos moleculares geram aumento da viscosidade(GUDMUNDSSON e HAFSTEINSSON, 1997).

$\mathrm{Na}$ tabela 2 observa-se que a solução de gelatina apresentou viscosidade de 1,13 cP, mostrando-se semelhante aos valores descritos para outras espécies de pescado como a corvina (Micropogonias furnieri) 1,68 cP (SCHIMITZ et. al., 2010), e megrim (Lepidorhombus boscii) 1,0 cP (MONTERO e GÓMEZ-GUILLÉN, 2000). 
A gelatina obtida de ossos de bagre apresentou viscosidade de 0,66 cP. Esse valor é inferior aos apresentados por Bandeira (2009), que relatou valores de 0,8 a 1,4 cP para gelatina de ossos de carpa. Essa diferença pode ser explicada pelas condições e/ou processo empregados na extração da gelatina, ou ainda pela utilização de diferentes espécies de pescado.

Tabela 2 - Propriedades viscoelásticas de gelatina extraída da pele e ossos de bagre.

\begin{tabular}{ccc}
\hline Gelatina & Pele & Ossos \\
\hline Viscosidade $(\mathrm{cP})$ & 1,13 & 0.66 \\
Ponto de Fusão $\left({ }^{\circ} \mathrm{C}\right)$ & $22,16 \pm 0,58$ & $21 \pm 0,99$ \\
\hline
\end{tabular}

Valores médios referentes a três determinações \pm desvio padrão.

A temperatura de fusão média da gelatina de pele de bagre foi de $22,16{ }^{\circ} \mathrm{C}$. Valor acima dos citados por e Gómez-Guillén et al.,(2002) que analisaram gelatinas de duas espécies de água quente - megrim e linguado, as quais apresentaram temperaturas de fusão em torno de $21{ }^{\circ} \mathrm{C}$. Alfaro e Silva (2010) relataram ponto de fusão para gelatinas extraídas de pele de tilápia (Oreochromis niloticus) em torno de $27^{\circ} \mathrm{C}$, valor consideravelmente superior aos encontrados para gelatina de pele de bagre africano.

Para a gelatina extraída de ossos o valor é $21{ }^{\circ} \mathrm{C}$ sendo inferior aos valores citados por Bandeira (2009) que apresenta valores entre 23 a $26{ }^{\circ} \mathrm{C}$ para gelatinas extraídas de ossos da cabeça de carpa. Alfaro et al. (2009) relatou superior ponto de fusão para gelatina de ossos de pescadafoguete (Macrodon ancylodon), com valor em torno de $23,5^{\circ} \mathrm{C}$.

O ponto de fusão mais elevado caracteriza melhores propriedades físicas e indica a possibilidade de obtenção de gelatina com propriedades mais similares às obtidas de mamíferos, isto provavelmente se deve à quantidade de iminoácidos presentes na gelatina. Os valores de ponto de fusão são diretamente influenciados pela origem da matéria prima ou ainda o processo de extração.

\section{Conclusão}

O rendimento do processo de extração da gelatina de pele foi superior a de ossos de bagre africano. As gelatinas de pele e ossos apresentaram valor de $\mathrm{pH}$ semelhantes e pontos de fusão de 22,16 e $21{ }^{\circ} \mathrm{C}$, respectivamente. A gelatina de pele possui viscosidade superior a de ossos, com valor semelhante aos descritos para outras espécies de pescado.

\footnotetext{
Abstract

This study aimed to produce gelatin skin and bones of catfish (Clarias gariepinus) and determine the viscosity, melting point, process yield and $\mathrm{pH}$ of the gelatin extracted. To obtain the gelatin raw materials were subjected to pre-treatment alkaline and acid, with subsequent extraction for 12 hours at $45^{\circ} \mathrm{C}$. The viscosity of gelatin skin was $1.13 \mathrm{cP}$ and bones $0.66 \mathrm{cP}$. The value of the
} 
melting point of gelatin skin was $22.16^{\circ} \mathrm{C}$ while for gelatin bones $21{ }^{\circ} \mathrm{C}$. The skin gelatin showed a process yield (5.85\%) considerably greater than that of bone (1.18\%). The pH of the gelatins was similar.

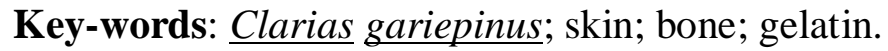

\section{Referências}

ALFARO A. T.; Otimização do processo e determinação das propriedades funcionais da gelatina de ossos de pescado. Rio Grande, 2004. Dissertação de Mestrado em Engenharia e Ciências de Alimento, Fundação Universidade Federal do Rio Grande.

ALFARO, A.T.; FONSECA, G.G.; COSTA, C.S.; PRENTICE, C. Effect of extraction parameters on the properties of gelatin from King weakfish (Macrodon ancylodon) bones. Food Science and Technology International, v. 15, p. 553562, 2009. DOI: 10.1177/1082013209352921

ALFARO AT, SILVA EF. Propriedades reológicas da gelatina obtida a partir de pele de tilápia (Oreochromis niloticus). Rev Inst Adolfo Lutz. São Paulo, 2010; 69(4):555-61.

A.O.A.C. Official methods and recommended practices of the american oilchemist's society. Washington: D. Feistane, 1995.

BANDEIRAS, S.F. Extração e caracterização da gelatina obtida de cabeça de carpas ( Aristichthys mobilis). Rio Grande, 2004. Dissertação de Mestrado, Engenharia e Ciências de Alimento, Fundação Univercidade Federal do Rio Grande, 2004.

BADII, F.; HOWELL, N. K. Fish gelatin: Structure, gelling properties and interaction with egg albumen proteins. Food Hydrocolloids, v. 20, p. 630-640, 2006. DOI: 10.1016/j.foodhyd.2005.06.006

B.S.I. (British Standards Institution) Methods for sampling and testing gelatin (physical and chemical methods). London. 1975.

CHOI, S. S.; REGENSTEIN, J.M. Physicochemical and sensory characteristics of fish gelatin. Journal of Food Science. v. 65, p. 194-199, 2000. DOI: 10.1111/j.1365-2621.2000.tb15978.x

KARIM, A.A. e BHAT, RAJEEV. Fish gelatin: properties, challenges, and prospects as an alternative to mammalian gelatins. Food Hydrocolloids, v. 23, p.563-576, 2009. DOI: 10.1016/j.foodhyd.2008.07.002

KOŁODZIEJSKA, I.; SK IERKA, E.; SADOWSKA, M.; KOŁODZIEJSKI, W.; NIECIKOWSKA, C. Effect of extracting time and temperature on yield of gelatin from different fish offal. Food Chemistry, p. 700-706, 2009.

GIMÉNEZ, B GOMÉZ-GUILLÉN, M. C.MONTERO, P. The role of salt washing of fish skins in chemical and rheological properties of gelatin extracted. Institute del Frío (CSIC), Ciudad Universitaria, 28040 Madrid, Spain 2004.

GÓMEZ-GUILlÉN, M. C.; TURNAY, J.; FERNÁNDEZ-DÍAZ, M. D.; ULMO, N.; LIZARBE, M. A.; MONTERO, P. Structural and physical properties of gelatin extracted from different marine species: a comparative study. Food Hydrocolloids, v. 16, p. 25 - 34, 2002. DOI: 10.1016/S0268-005X(01)00035-2

GUDMUNDSSON, M.; HAFSTEINSSON, H. Gelatin from cod skins as affected by chemical treatments. Journal of Food Science, v. 62, p. 37-39, 1997. DOI: 10.1111/j.1365-2621.1997.tb04363.x

HAUG, I. J.; DRAGET, K. I.; SMIDSROD, O. Physical and rheological properties of fish gelatin compared to mammalian gelatin. Food Hydrocolloids, v. 18, p. 203-213, 2004. DOI: 10.1016/S0268-005X(03)00065-1

IBAMA. Instituto Brasi leiro do Meio Ambiente e dos Recursos Naturais Renováveis. Estatística da pesca - grandes regiões e unidades da federação / Boletim, Brasília: Ibama, 2008.

JAMILAH, B.; HARVINDER, K. G. Properties of gelatins from skins of fish: black tilapia (Oreochromis mossambicus) and red tilapia (Oreochromis nilotica). Food Chemistry, v. 77, p. 81-84, 2002. DOI: 10.1016/S0308-8146(01)00328-4 
MONTERO, P.; GÓMEZ-GUILLÉN, M. C. Extracting conditions for megrim (Lepidorhombus boscii) skin collagen affect functional properties of the resulting gelatin. Food Chemistry and Toxicology. v. 65, n. 3, p. $434-438,2000$

MUYONGA, J. H.; COLE, C. G. B.; DUODU, K. G. Extraction and physicochemical characterisation of Nile perch (Lates niloticus) skin and bone gelatin. Food Hydrocolloids, v. 18, p. 581-592, 2004. DOI: 10.1016/j.foodhyd.2003.08.009

SCHMITZ, V. U.; BANDEIRA, S. F.; ESQUERDO, V. M. et. al. Propriedades físicas de gelatinas obtidas a partir de cabeças de corvina. XII ENPOS - II Mostra Científica. Universidade Federal do Rio Grande, RS, 4 p., 2010.

SOUZA, M. L. R.; SILVIA LIMA, FURUYA, W. M.; PINTO, ADRIANA A.B.; LOURES, T. R. R.;E POVH, J. A. Estudo de carcaça do bagre africano (Clarias gariepinus) em diferentes categorias de peso.. Departamento de Zootecnia, Universidade Estadual de Maringá, Maringá-Paraná, Brasil. Acta Scientiarum. v. 21(3):637-644, 1999.

WANG, LIN; AN, XINXIN; YANG, FANGMEI; XIN, ZHIHONG; ZHAO, LIYAN; HU, QIUHUI. Isolation and characterisation of collagens from the skin, scale and bone of deep-sea redfish (Sebastes mentella). Food Chemistry, v. 108, p. 616-623, 2008. DOI: 10.1016/j.foodchem.2007.11.017

YANG, H.; WANGA, Y.; ZHOUC, P.; REGENSTENIN, J. M. Effects of alkaline and acid pretreatment on the physical properties and nanostructures of the gelatin from channel catfish skin.s, Food Hydrocolloids. v. 22, p 1541-1550, 2008. DOI: 10.1016/j.foodhyd.2007.10.007

Trabalho selecionado para apresentação oral durante a VIII SETAL- Semana de Tecnologia de Alimentos- Câmpus Ponta Grossa- Universidade Tecnológica Federal do Paraná- 01 a 03 de junho de 2011. Suplemento especial da RBTA. 